\title{
Application of a new methodology and $R$ package reveals a high burden of healthcare-associated infections (HAI) in Germany compared to the average in the European Union/European Economic Area, 2011 to 2012
}

Benedikt Zacher ${ }^{1,2}$, Sebastian Haller ${ }^{1,2}$, Niklas Willrich ${ }^{1}$, Jan Walter ${ }^{1}$, Muna Abu Sin ${ }^{1}$, Alessandro Cassini ${ }^{3}$, Diamantis Plachouras Carl Suetens $^{3}$, Michael Behnke ${ }^{4}$, Petra Gastmeier ${ }^{4}$, Lothar H. Wieler ${ }^{1}$, Tim Eckmanns ${ }^{1}$

1. Robert Koch Institute, Berlin, Germany

2. These authors contributed equally to this work

3. European Centre for Disease Prevention and Control, Stockholm, Sweden

4. Charité Universitätsmedizin Berlin, Berlin, Germany

Correspondence: Benedikt Zacher (ZacherB@rki.de)

Citation style for this article:

Zacher Benedikt, Haller Sebastian, Willrich Niklas, Walter Jan, Abu Sin Muna, Cassini Alessandro, Plachouras Diamantis, Suetens Carl, Behnke Michael,

Gastmeier Petra, Wieler Lothar H., Eckmanns Tim. Application of a new methodology and R package reveals a high burden of healthcare-associated infection (HAl) in Germany compared to the average in the European Union/European Economic Area, 2011 to 2012. Euro Surveill. 2019;24(46):pii=1900135. https://doi. org/10.2807/1560-7917.ES.2019.24.46.1900135

Background: Healthcare-associated infections (HAIs) pose a major challenge to health systems. Burden of disease estimations in disability-adjusted life years(DALYs) are useful for comparing and ranking HAls. Aim: To estimate the number of five common HAls, their attributable number of deaths and burden for Germany. Methods: We developed a new method and $R$ package that builds on the approach used by the Burden of Communicable Diseases in Europe (BCoDE) project to estimate the burden of HAls for individual countries. We used data on healthcareassociated Clostridioides difficile infection, healthcareassociated pneumonia, healthcare-associated primary bloodstream infection, healthcare-associated urinary tract infection and surgical-site infection, which were collected during the point prevalence survey of HAls in European acute-care hospitals between 2011 and 2012. Results: We estimated 478,222 (95\% uncertainty inter$\operatorname{val}(\mathrm{UI}): 421,350-537,787)$ cases for Germany, resulting in $16,245(95 \% \mathrm{UI}: 10,863-22,756)$ attributable deaths and $248,920(95 \%$ UI: $178,693-336,239)$ DALYs. Despite the fact that Germany has a relatively low hospital prevalence of HAls compared with the European Union/European Economic Area(EU/EEA) average, the burden of HAls in Germany (308.2 DALYs/100,000 population; 95\%UI:221.2-416.3) was higher than the EU/EEA average (290.0 DALYs/100,000 population; $95 \%$ UI: 214.9-376.9). Our methodology is applicable to other countries in or outside of the EU/EEA. An R package is available from https://CRAN.R-project.org/ package $=$ BHAl.
Conclusion: This is the first study to estimate the burden of HAls in DALYs for Germany. The large number of hospital beds may be a contributing factor for a relatively high burden of HAls in Germany. Further focus on infection prevention control, paired with reduction of avoidable hospital stays, is needed to reduce the burden of HAls in Germany.

\section{Introduction}

Healthcare-associated infections(HAls) are associated with an increased risk in morbidity, mortality and excess healthcare costs. It is estimated that 20 to $30 \%$ of HAls in Germany could be preventable primarily through improved adherence to hygiene recommendations and optimisation of procedures [1]. Increasing adherence and changing behaviour of clinical personnel is resource-intensive, and resources for prevention are limited [2]. Estimates of health burden of HAls are therefore needed to assess their relevance compared with other communicable diseases and help with evidence-based prioritisation. Since a patient with a $\mathrm{HAl}$ experiences this alongside the primary reason for being in a healthcare setting, attributing complications and death to a HAl is particularly challenging.

The European Centre for Disease Prevention and Control (ECDC) published the first estimates of the health burden attributable to HAIs in the European Union and European Economic Area (EU/EEA) in 2016 [3]. It used the same incidence-based approach as the ECDC Burden of Communicable Diseases in 


\section{FIGURE 1}

Overview of the workflow of the estimation of the burden of healthcare-associated infections implemented in the BHAI R package
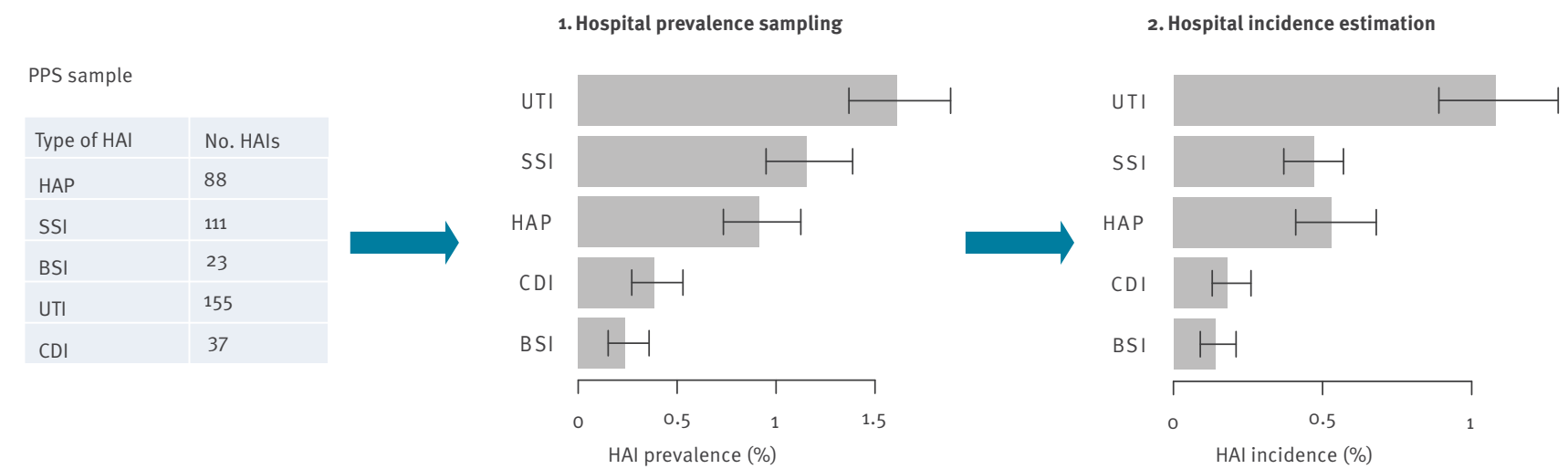

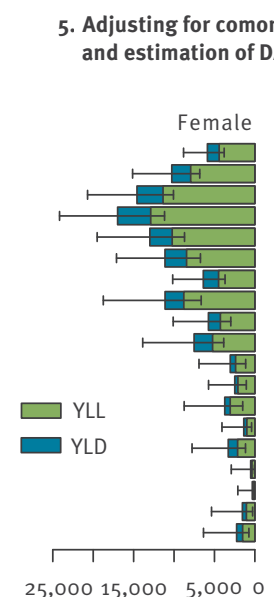

No. DALYS

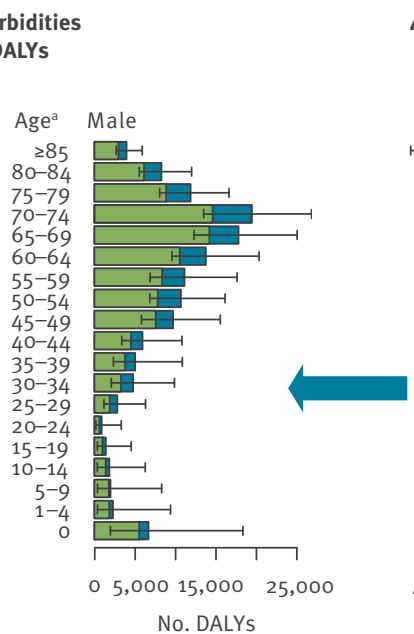

No. DALYS

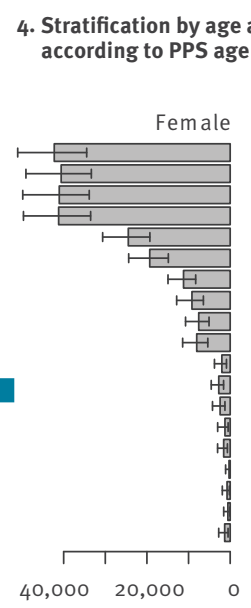

No. HAls

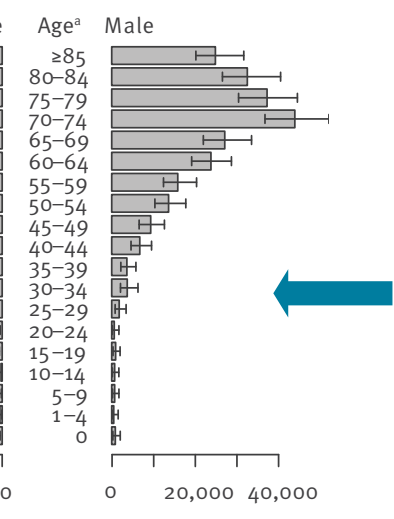

No. HAls

BHAI: Burden of Healthcare-Associated Infections; BSI: healthcare-associated primary bloodstream infection; CDI: healthcare-associated Clostridioides difficile infection; DALY: disability-adjusted life year; HAI: healthcare-associated infection; HAP: healthcare-associated pneumonia; No.: Number of; PPS: point prevalence survey; SSI: surgical site infection; UTI: healthcare-associated urinary tract infection; YLD: years lived with disability; YLL: years of life lost.

age in years.

The error bars indicate the $95 \%$ uncertainty intervals.

Europe (BCoDE) project to estimate the number of disability-adjusted life years (DALYs) due to HAls. The estimates were based on data from the ECDC point prevalence survey (PPS) of HAls and antimicrobial use in European acute care hospitals between 2011 and 2012 [4-6]. DALYs are a comprehensive measure to rank the burden of diseases and this way of ranking is more valuable than ranking only on incidence or prevalence. The BCoDE tool assists in calculating the number of DALYs specifically for a number of communicable diseases, including HAls [7].

In this study, we used a new adapted methodology to estimate the incidence of HAls, attributable deaths and DALYs in 2011 in Germany for five common types of HAls: healthcare-associated Clostridioides difficile infection(CDI), healthcare-associated pneumonia(HAP), healthcare-associated primary bloodstream infection(BSI), healthcare-associated urinary tract infection(UTI) and surgical site infection (SSI). In addition, we compared our estimates of the burden of HAls in Germany with estimates for the EU/EEA.

\section{Methods}

Study population and study design

The German PPS data used in this study were collected in 2011 as part of the ECDC PPS, which was conducted between 2011 and 2012 in 29 EU/EEA countries and 
Annual burden of five types of healthcare-associated infections, German point prevalence survey sample, Germany, 2011

\begin{tabular}{|c|c|c|c|c|c|c|}
\hline $\begin{array}{l}\text { Type } \\
\text { of HAI }\end{array}$ & Sample & $\begin{array}{c}\text { Number of HAls } \\
\text { Point estimate }(95 \% \mathrm{UI})\end{array}$ & $\begin{array}{l}\begin{array}{c}\text { Number of attributable } \\
\text { deaths }\end{array} \\
\text { Point estimate }(95 \% \text { UI) }\end{array}$ & $\begin{array}{c}\text { Number of DALYs } \\
\text { Point estimate }(95 \% \text { UI) }\end{array}$ & $\begin{array}{c}\text { Number of YLLs } \\
\text { Point estimate }(95 \% \text { UI) }\end{array}$ & $\begin{array}{c}\text { Number of YLDs } \\
\text { Point estimate }(95 \% \text { UI) }\end{array}$ \\
\hline HAP & $\begin{array}{c}\text { German } \\
\text { PPS }\end{array}$ & $106,586(83,618-137,476)$ & $3,968(1,107-8,164)$ & $69,508(34,042-117,232)$ & $41,306(11,475-84,483)$ & $27,539(16,528-42,824)$ \\
\hline SSI & $\begin{array}{c}\text { German } \\
\text { PPS }\end{array}$ & $93,222(75,369-114,241)$ & $2,328(1,888-2,882)$ & $28,842(23,313-35,303)$ & $28,376(22,983-34,714)$ & $452(352-580)$ \\
\hline BSI & $\begin{array}{c}\text { German } \\
\text { PPS }\end{array}$ & $26,976(16,520-42,252)$ & $3,905(2,004-6,987)$ & $58,350(30,940-104,227)$ & $49,578(25,499-90,816)$ & $8,787(4,463-16,609)$ \\
\hline UTI & $\begin{array}{c}\text { German } \\
\text { PPS }\end{array}$ & $214,150(175,086-253,524)$ & $3,664(1,462-7,533)$ & $66,701(27,890-128,543)$ & $44,871(18,043-92,915)$ & $20,243(8,095-40,522)$ \\
\hline CDI & $\begin{array}{c}\text { German } \\
\text { PPS }\end{array}$ & $36,002(25,108-49,934)$ & $1,917(112-4,547)$ & $20,890(2,023-49,443)$ & $19,937(1,166-47,973)$ & $977(172-2,125)$ \\
\hline All & $\begin{array}{c}\text { German } \\
\text { PPS }\end{array}$ & $478,222(421,350-537,787)$ & $16,245(10,863-22,756)$ & $248,920(178,693-336,239)$ & $190,245(131,301-264,573)$ & $59,076(40,263-84,578)$ \\
\hline
\end{tabular}

BSI: healthcare-associated primary bloodstream infection; CDI: healthcare-associated Clostridioides difficile infection); DALY: disabilityadjusted life year; HAI: healthcare-associated infection; HAP: healthcare-associated pneumonia; PPS: point prevalence survey; SSI: surgical site infection; UI: uncertainty interval; UTI: healthcare-associated urinary tract infection; YLD: years lived with disability; YLL: years of life lost.

a This is the median.

Croatia, which has since become the $28^{\text {th }}$ EU Member State [6]. Three datasets were available for our analyses:

(i) The representative German PPS sample consists of 46 hospitals, randomly selected from a list of all German hospitals stratified by hospital size [6]. The sample includes 9,626 patients in total.

(ii) A larger German convenience sample of the same PPS, which is not representative by hospital size, consists of 132 hospitals and 41,539 patients.

(iii) For comparison, we used the ECDC PPS sample, which includes 273,753 patients from 1,149 hospitals in 29 EU/EEA countries in 2011 and 2012 and Croatia [6]. We estimated the burden of HAls for the ECDC PPS sample using the original data and our adapted methodology, which is implemented in the Burden of Healthcare-associated Infections (BHAI) R package (see below for more details).

The types of HAls selected for this study were as described by Cassini et al. [3]. The selection was based on prevalence, availability of data and evidence from systematic reviews. HAls were defined according to EU case definitions [8], with the exception of CDIs, for which we selected a syndrome-based approach.

\section{Outcome measure}

DALYs are a composite measure of years lived with disability (YLDs) and years of life lost (YLLs) accounting for incidence, severity and mortality of the disease simultaneously. This approach enables ranking and comparability of the health burden of different types of HAls in one metric.

\section{Outcome trees with transition probabilities}

Disease models or outcome trees illustrate progression pathways of HAls over time, starting with acute infection and ending with either recovery, permanent disability, or death. To take into account all possible health consequences of HAls, outcome trees were developed based on 13 systematic reviews of the literature $[9,10]$. Attributable transition probabilities were extracted from the literature to avoid overestimation of the health burden due to comorbidities, which is a particular risk in patients with an HAl. Health outcomes were related to each other by a transition probability and each outcome included a duration and a disability weight $[9,11]$. We used the same outcome trees with transition probabilities and disability weights, which were used by Cassini et al.. Detailed descriptions of the outcome trees can be found in the supplement of Cassini et al. or within the ECDC BCoDE toolkit, in which the disease model parameters are described in detail $[3,7]$. The systematic reviews and results of meta-analyses were published in a separate document [9].

\section{Workflow of the Burden of Healthcare-}

Associated Infections (BHAI) methodology

Our approach was implemented by the BHAI R package, which performs a predefined number (default: 1,000) of Monte Carlo simulations (Figure 1).

Step 1: estimation of hospital prevalence of healthcareassociated infections

For each type of HAI, hospital prevalence (P) was estimated using the observed number of patients with an HAl and the total number of patients in the PPS. Prevalence was calculated separately for the German representative and convenience samples. Uncertainties were taken into account by sampling from a mixture 


\section{FIGURE 2}

Annual number of healthcare-associated infections plotted against the annual number of attributable deaths for five types of healthcare-associated infections ${ }^{\mathrm{a}}$, Germany, 2011

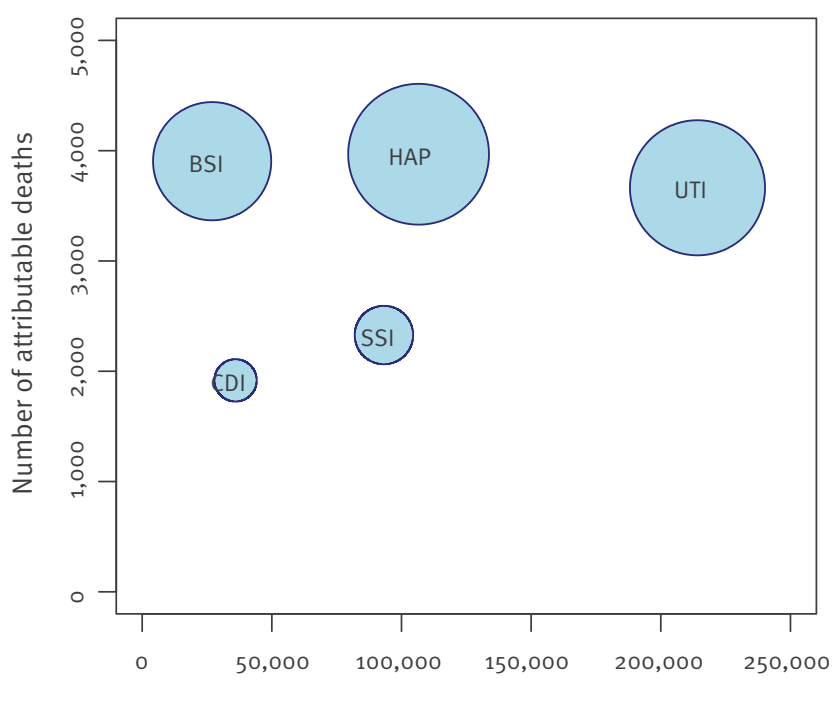

Number of HAls

BSI: healthcare-associated primary bloodstream infection; CDI: healthcare-associated Clostridioides difficile infection; DALY: disability-adjusted life year; HAI: healthcare-associated infection; HAP: healthcare-associated pneumonia; SSI: surgical site infection; UTI: healthcare-associated urinary tract infection.

a Data are based on the 2011 German point prevalence study sample.

The diameter of the bubbles is proportional to the annual numbers of DALYs, Germany, 2011.

of two beta distributions, which form the exact midP-Clopper-Pearson confidence interval $(\mathrm{Cl})$ for proportions. The use of this mixed distribution gives uncertainty intervals $(2.5 \%$ and $97.5 \%$ quantiles) that accurately reflect exact $\mathrm{Cls}$.

Step 2: estimation of hospital incidence of healthcareassociated infections using prevalence

We estimated hospital incidence (I) from P for each type of $\mathrm{HAl}$ using a modification of the Rhame-Sudderth formula: $I=P \times L A / L O I$, where $L A$ is the mean length of stay and LOI is the length of infection [12]. The average LA of all patients in the survey was calculated from data of participating hospitals, based on the number of discharges and patient days in the previous year [13]. The modified Rhame-Sudderth formula defines LOI as the total time of infection. Often, only the time from infection onset until the date of survey $\left(\mathrm{LOI}_{\mathrm{pps}}\right)$ is collected. Estimators of LOI that have been used in the past are the median or mean of $\mathrm{LOI}_{\mathrm{pps}}$, which are approximations for the true LOI. We used the Grenander estimator which was employed in 2018 to estimate the number of HAls in the EU/EEA $[14,15]$. In this method, the distributions of $\mathrm{LOI}_{\mathrm{pps}}$ were estimated from the PPS data assuming monotonicity. This assumption is justified by the timeline of occupancy of a hypothetical bed: There will always be more patients on the first day than on the second day of hospitalisation, and more on the second day than the third day, etc. The average proportion of patients with a HAl on the day of the PPS was derived from the following distribution: Probability $\left(\mathrm{LOI}_{\mathrm{pps}}=1\right)$. The expected LOI of a HAI was then estimated as $1 /$ Probability $\left(\mathrm{LOI}_{\mathrm{pps}}=1\right)$. One caveat of this method is that it requires a large sample size. However, Willrich et al. showed that the mean, which is biased but more robust with small samples, performed well in most applications [14]. Our methodology therefore used a weighted sum of the mean of LOI ${ }_{\text {pps }}$ and the Grenander estimator using the sigmoid function. This allowed the influence of the Grenander estimator to become larger, and therefore the estimates were more accurate with larger sample sizes. Simulations that analyse the robustness of the LOI estimates for different sample sizes using this new approach are summarised in Supplementary Figure S1. BHAl takes into account the uncertainty about the LOI estimate using bootstraps in each Monte Carlo simulation. The larger German PPS convenience sample was used for more reliable estimation of LOIs. The LOIs used for computations are displayed in Supplementary Figure S2.

Step 3: from hospital incidence to population incidence of healthcare-associated infections

Hospital incidences of HAls per patient were extrapolated to the whole population by multiplying with the number of hospital discharges in Germany. The population data and number of hospital discharges for 2011 were obtained from the Eurostat database (http:// ec.europa.eu/eurostat/ ) for all countries following the approach of Cassini et al. [3,13].

Step 4: stratification by age and sex according to point prevalence survey age distribution

After estimating the total annual number of cases in the previous steps, BHAl was used to estimate the number of attributable deaths and DALYs. For DALY calculations, HAls distributed according to age and sex were needed to account for different life expectancies. For each type of $\mathrm{HAl}$, the age and sex distributions were obtained from the ECDC PPS. With smaller countrywide PPS, the stratified number of cases may exhibit many empty strata (without cases). We used Bayesian analysis to include prior knowledge about the distribution and combined this information with the evidence from the observed data in a posterior distribution of the true parameters.

The posterior distribution was a multinomial likelihood with a Dirichlet prior distribution, which is parameterised by prior weights. The estimation of the health burden from the German PPS sample was conducted by applying the number of cases from the German convenience sample as prior weights. This distribution also contained empty strata, although fewer than in the German PPS sample. Since all prior weights had to be > o, the BHAI R package added a small pseudocount to all strata: $0.001 \times$ sum(prior weights). In each Monte 
Annual burden per 100,000 population of five types of healthcare-associated infections, German PPS sample, German convenience sample and ECDC PPS sample, Germany, EU/EEA, 2011-2012

\begin{tabular}{|c|c|c|c|c|c|c|c|}
\hline $\begin{array}{l}\text { Annual burden } \\
\text { measure }\end{array}$ & Sample & $\begin{array}{c}\text { HAP } \\
\text { Point estimate }^{\mathrm{a}} \\
(95 \% \mathrm{UI})\end{array}$ & $\begin{array}{c}\text { UTI } \\
\text { Point estimate }{ }^{\mathrm{a}} \\
(95 \% \text { UI })\end{array}$ & $\begin{array}{c}\text { BSI } \\
\text { Point estimate }^{\mathrm{a}} \\
(95 \% \mathrm{UI})\end{array}$ & $\begin{array}{c}\text { SSI } \\
\text { Point estimate }^{a} \\
(95 \% \text { UI })\end{array}$ & $\begin{array}{c}\text { CDI } \\
\text { Point estimate } \\
(95 \% \text { UI })\end{array}$ & $\begin{array}{c}\text { All } \\
\text { Point estimate }^{\mathrm{a}} \\
(95 \% \mathrm{UI})\end{array}$ \\
\hline \multirow{3}{*}{$\begin{array}{l}\text { HAls per } \\
100,000\end{array}$} & German PPS & $132.0(103.5-170.2)$ & $265.1(216.8-313.9)$ & $33.4(20.5-52.3)$ & $115.4(93.3-141.4)$ & $44.6(31.1-61.8)$ & $592.1(521.7-665.8)$ \\
\hline & $\begin{array}{c}\text { German } \\
\text { convenience }\end{array}$ & $162.3(137.5-190.7)$ & $228.7(200-260.7)$ & $52.7(42-66.9)$ & $146.9(126.5-167.8)$ & $44.5(35.6-55.4)$ & $636.1(586.7-689.2)$ \\
\hline & $\begin{array}{c}\text { ECDC PPS (EU/ } \\
\text { EEA) }\end{array}$ & $143.7(136.9-150.8)$ & $174.7(166.3-182.4)$ & $22.2(20-25.1)$ & $111.3(105.4-116.6)$ & $16.0(14.2-18.3)$ & $467.9(456.2-480.2)$ \\
\hline \multirow{3}{*}{$\begin{array}{l}\text { Attributable } \\
\text { deaths per } \\
100,000\end{array}$} & German PPS & $4.9(1.4-10.1)$ & $4.5(1.8-9.3)$ & $4.8(2.5-8.7)$ & $2.9(2.3-3.6)$ & $2.4(0.1-5.6)$ & $20.1(13.4-28.2)$ \\
\hline & $\begin{array}{c}\text { German } \\
\text { convenience }\end{array}$ & $6.1(1.4-11.7)$ & $3.9(1.6-8)$ & $7.9(4.7-11.8)$ & $3.7(3.2-4.2)$ & $2.5(0.1-5.3)$ & $24.4(17.2-32.6)$ \\
\hline & $\begin{array}{c}\text { ECDC PPS (EU/ } \\
\text { EEA) }\end{array}$ & $5.3(1.3-10.2)$ & $3.0(1.2-5.9)$ & $3 \cdot 3(2.1-4.6)$ & $2.6(2.4-2.7)$ & $0.9(0-1.8)$ & $15.3(10.2-21.2)$ \\
\hline \multirow{3}{*}{$\begin{array}{l}\text { DALYs per } \\
100,000\end{array}$} & German PPS & $86.1(42.1-145.1)$ & $82.6(34.5-159.2)$ & $72.2(38.3-129)$ & $35.7(28.9-43.7)$ & $25.9(2.5-61.2)$ & $308.2(221.2-416.3)$ \\
\hline & $\begin{array}{c}\text { German } \\
\text { convenience }\end{array}$ & $103.4(51.5-166.5)$ & $69.5(29.9-127.7)$ & $113.5(72.2-166)$ & $45.0(38.8-51.3)$ & $26.5(2.5-55.6)$ & $359.3(266.6-461.5)$ \\
\hline & $\begin{array}{c}\text { ECDC PPS (EU/ } \\
\text { EEA) }\end{array}$ & $109.8(55.3-170.5)$ & $57.1(24 \cdot 3-102.9)$ & $76.2(52.6-104.8)$ & $35.1(33.3-36.8)$ & $10.0(0.9-19.2)$ & $290.0(214.9-376.9)$ \\
\hline
\end{tabular}

BSI: healthcare-associated primary bloodstream infection; CDI: healthcare-associated Clostridioides difficile infection; DALY: disabilityadjusted life year; ECDC: European Centre for Disease Prevention and Control; EU/EEA: European Union/European Economic Area; HAP: healthcare-associated pneumonia; PPS: point prevalence survey; SSI: surgical site infection; UTI: healthcare-associated urinary tract infection.

a This is the median.

Carlo simulation, a sample was drawn from the posterior distribution, which incorporated uncertainty about the age and sex distribution. The sampled age and sex probability distribution was then multiplied with the overall annual number of cases to calculate the stratified number of cases for each type of HAI.

Step 5: adjusting for different life expectancies

The McCabe score, documented in the ECDC PPS between 2011 and 2012, gave a reference point for the life expectancy of a patient according to severity of underlying disease. It allowed patients to be stratified in three groups according to whether the underlying disease was (i) non-fatal (normal life expectancy), (ii) ultimately fatal (average expected life expectancy of 3 years), or(iii) rapidly fatal (average expected life expectancy of 0.5 years). German PPS data were collected using the unit-based ('light') protocol of the ECDC PPS, for which McCabe scores are not recorded [6]. We applied the McCabe score distribution of the ECDC PPS to Germany(i.e. cumulative data from all countries that collected McCabe scores with the standard protocol), assuming that the McCabe score distribution in Germany would be comparable to that of the entire EU/EEA. In the BHAI R package, we calculated a probability distribution of $\mathrm{McC}$ abe scores for each $\mathrm{HAl}$, age and sex stratum and distributed cases by multiplication with the number of estimated cases in each simulation. In each Monte Carlo simulation, parameters of disease outcome trees, i.e. disability weights, durations and transition probabilities, were sampled for the whole population. The number of fatalities attributable to each type of HAI was calculated by multiplying the number of cases with the probability of death. The number of DALYs was calculated with respect to the remaining life expectancy in each stratum.

\section{Validity of estimates obtained with the Burden of Healthcare-Associated Infections (BHAI) method}

We simulated ECDC PPS data ranging from 1,000 to 200,000 surveyed patients (the latter being approximately equal to the size of the ECDC PPS sample). This allowed us to compare the sampling approach implemented in the BHAI R package with the approach of the previous burden estimation using the BCoDE toolkit $[3,7]$. In each simulation, given the number of surveyed patients, the prevalence for each type of HAl from the overall ECDC PPS sample was used to simulate the number of cases of each type of HAI. These were then randomly distributed to strata according to the age, sex and McCabe distributions of the ECDC PPS. The length of the HAls was sub-sampled according to the number of HAls in each simulation, while the mean LA was kept fixed to the estimate from the full sample. In order to evaluate the effect of the sample size on the estimation, the results from the simulated data were then compared with the results from the ECDC PPS sample.

\section{Availability}

Our method was implemented by the BHAI R package, which is freely available from https://cran.r-project. org/ and as a Supplement to this paper (Supplement S1). The data and analysis code used for calculating the German and EU/EEA burden of HAls are attached to the package, which can be used to reproduce the analysis and the results described in this study. 
Annual burden of five types of healthcare-associated infections with (A) attributable deaths per 100,000 population and (B) disability-adjusted life years per 100,000 population, Germany, EU/EEA, 2011-2012

A.

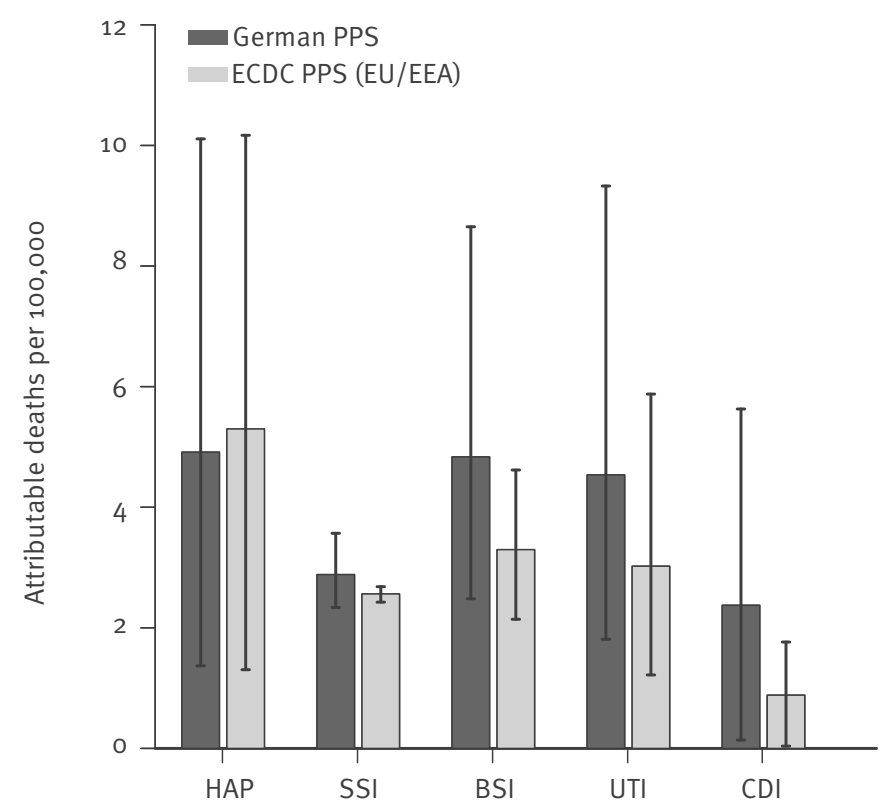

B.

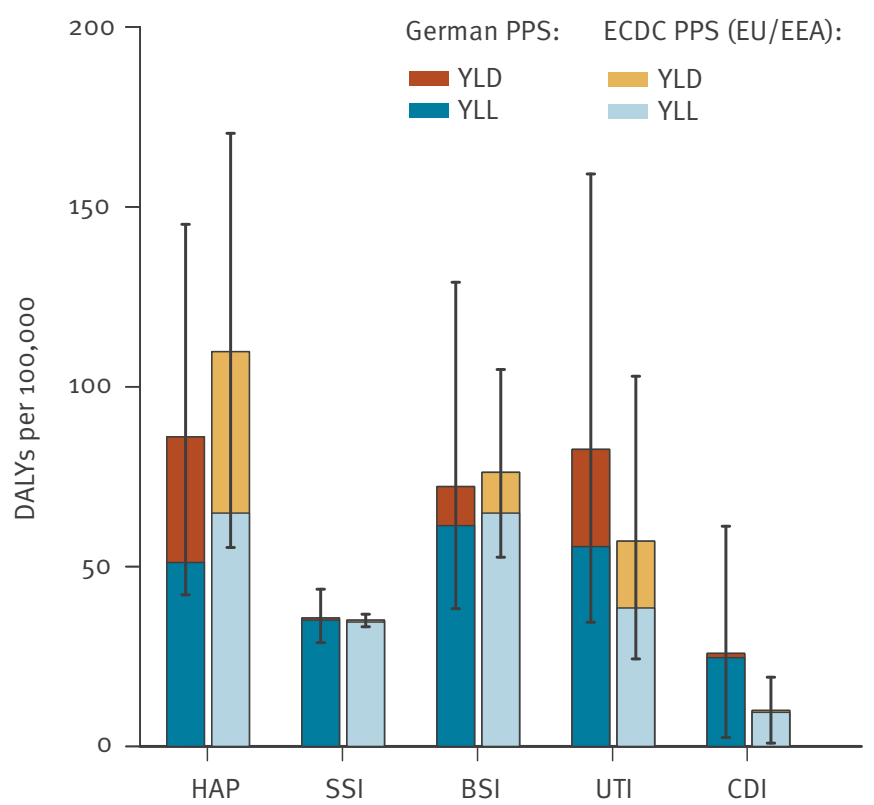

BSI: healthcare-associated primary bloodstream infection; CDI: healthcare-associated Clostridioides difficile infection; DALY: disabilityadjusted life year; ECDC: European Centre for Disease Control and Prevention; EU/EEA: European Union/European Economic Area; HAI: healthcare-associated infection; HAP: healthcare-associated pneumonia; PPS: point prevalence survey; SSI: surgical site infection; UI: uncertainty interval; UTI: healthcare-associated urinary tract infection; YLD: years lived with disability; YLL: years of life lost.

For all five HAIs, attributable deaths amount to 20.1 per 100,000 population (95\% Ul: 13.4-28.2) and DALYs to 308.2 per 100,000 population (95\% UI: 221.2-416.3).

The error bars indicate the $95 \%$ UI.

\section{Ethical statement}

This study was based on health information collected and published within the ECDC PPS and did not require informed consent from participants. Reported infectious disease data were provided in aggregated format by specific age and sex strata, without personal identifiers.

\section{Results}

\section{Burden of healthcare-associated infections in Germany}

Using German PPS data from 2011, we estimated that there were $478,222(95 \%$ UI: 421,350-537,787) new cases of the selected types of HAls, and 16,245 (95\% UI: 10,863-22,756) attributable deaths in Germany in that year(Table 1). The health burden of acute HAls and sequelae amounted to a median of 248,920 (95\%UI:178,693-336,239) DALYs, 59,076 (95\% UI: 40,263-84,578) YLDs and 190,245 (95\% Ul: 131,301-264,573) YLLs per year. Note that the sum of the median YLDs and YLLs does not exactly equal the median DALYs. This is because the sum of the median of two distributions (i.e. YLDs and YLLs for all 1,000 simulations) is not necessarily equal to the median of the sum of the distributions (i.e. DALYs calculated as YLD+YLL for each simulation). The relationship between incidence, mortality and DALYs is illustrated in Figure 2, which gives detailed information about the different impacts of HAls on population health. HAP and BSI accounted for $51 \%$ (127,858 DALYs/248,920 DALYs) of the burden of HAIs, but accounted for only $28 \%(133,562$ cases/478,222 cases) of the estimated number of HAls (Figure 2). UTIs accounted for $45 \%(214,150$ cases $/ 478,222$ cases $)$ of the cases, but for $27 \%(66,701$ DALYs/248,920 DALYS) of DALYs. Estimates of the length of the HAIs varied from 9 to 15 days depending on the type of HAI (Supplementary Figure S2).

From the larger German convenience sample, we estimated that there were 513,729 (95\% UI: 473,840-556,654) HAls per year, and 19,672 (95\%UI:13,921-26,310) attributable deaths, leading to 290,228 (95\% UI: 215,305-372,734) DALYs, 65,988 (95\% Ul: 46,856-89,194) YLDs and 224,939 (95\% UI: 160,973-298,122) YLLs (Supplementary Table S1, Supplementary Figure S3 and S4) in 2011. 
In both the German convenience and the representative PPS samples, $4.1 \%$ of patients in hospital (394 patients with $\mathrm{HAl} / 9,626$ patients and 1,688 patients with $\mathrm{HAl} / 41,539$ patients for each type of sample respectively) had at least one of the five types of HAls. The difference in disease burden found between the convenience and PPS samples was mainly due to an increased prevalence of HAPs (1.0\%; 424 patients with $\mathrm{HAP} / 41,539$ patients) and BSIs (0.34\%; 142 patients with $\mathrm{BSI} / 41,539$ patients) in the German convenience sample.

\section{Comparison to other communicable diseases}

From the German PPS sample, we estimated that the total burden of the five HAls in Germany was 308.2 DALYs per 100,000 population. In contrast, a previous study using the BCoDE methodology estimated that the burden of four other communicable diseases (influenza, Salmonella spp., hepatitis B and measles) in Germany was only 39.4 DALYs per 100,000 population (Supplementary Table S2) [16].

\section{Comparison to the European Union/European Economic Area}

From the ECDC PPS conducted between 2011 and 2012, we estimated the burden of HAls for the whole EU/EEA using the adapted BHAl method. We found that there were $2,365,466(95 \%$ Ul: $2,306,018-2,427,774)$ HAls, 77,483 (95\% UI: 51,502-106,982) attributable deaths and $1,465,822(95 \%$ UI: $1,086,252-1,905,451)$ DALYs in the EU/EEA (Supplementary Table S3).

Rates per 100,000 population are presented in Table 2 and allow for comparison of the three different samples. The incidence of HAls in Germany (German PPS representative sample) was 1.27 (95\% UI:1.14-1.43) times higher than the EU/EEA(ECDC PPS sample). The estimated number of attributable deaths per 100,000 population and DALYs per 100,000 population were 1.29 (95\% UI: $0.78-2.18$ ) and 1.06 (95\% UI: $0.68-1.67$ ) times higher in Germany (German PPS representative sample) than in the EU/EEA. This difference was even larger when comparing rates obtained from the German convenience sample with EU/EEA estimates. There was a 1.36 (95\% UI:1.25-1.47) higher incidence of HAls, 1.58 (95\% Ul:0.95-2.66) higher attributable mortality and 1.24 (95\% UI:0.83-1.84) higher DALY rate in Germany than in the EU/EEA. Comparisons of the rates obtained for Germany (German representative and convenience PPS samples) to the EU/EEA estimates(ECDC PPS sample) are given in Figures 3 and 4 and in Supplementary Figures $S_{5}$ and S6.

\section{Burden of Healthcare-Associated Infections (BHAI) methodology and $\mathrm{R}$ package}

The new methodology for the estimation of HAls presented in this study was implemented in the $R$ package BHAI (openly available from https://cran.r-project. org/). It was evaluated using a simulated PPS with sample sizes ranging from 1,000-200,000. This evaluation demonstrates the usability of BHAl for the estimation of the burden of HAls for large and small PPS samples (Supplementary Figure S7).

\section{Discussion}

In this study, we estimated the burden of HAls in Germany expressed in DALYs for the first time, based on point prevalence data from 2011. By considering incidence and severity of the disease simultaneously, we provided a more comprehensive view on the burden of HAls than previously available. This allowed for comparing and ranking the burden of HAls and other diseases in Germany.

The annual number of HAls in Germany was previously estimated between 400,000-600,000 cases and 10,000-15,000 attributable deaths per year. These estimates were based on data from the German PPS studies (NIDEP-1) from 1994 [17,18]. In the ECDC PPS, the annual number of HAls in Germany in 2011 was estimated at between $321,321-1,025,716$ [6]. It is important to note that different approaches and methods were used in these studies.

In this study, we estimated the numbers of cases for five types of HAl, associated fatalities and their health burden. These five types of $\mathrm{HAl}$ made up $79 \%$ of all HAls in the ECDC PPS sample, and the numbers of HAls in our study therefore do not represent the total number of HAls in the population. Nevertheless, our estimates of the number of HAls lie within the range of previous estimates, although the median estimates of the number of attributable deaths were higher.

In our study, BSIs had the lowest incidence of the five types of $\mathrm{HAl}$, but was responsible for a high number of DALYs due to its high attributable mortality. On the other hand, UTI, despite its high incidence, caused a comparable health burden since its attributable mortality was considerably lower. While, of all cases, the fatality rate of primary or secondary BSIs was 9-20\%, $5-20 \%$ of patients with UTI developed a secondary bacteraemia or urosepsis, which then led to death in $9-20 \%$ of the cases $[9,10]$. These shifts in ranking illustrate the importance of a comprehensive approach such as the burden estimation based on DALYs.

The health burden of HAls was substantially higher than the burden of other communicable diseases. For instance, the burdens of $\operatorname{HAP}(69,508 \mathrm{DALYs})$ and UTI $(66,701$ DALYs) were more than twice as large in comparison, and the burden of $\operatorname{BSI}(58,350$ DALYS $)$ was slightly less than twice as much as the burden of influenza(33,116DALYs) in Germany (Supplementary Table S2) [16]. The burden of all five considered HAls in Germany was 308.2 (95\% UI:221.2-416.3) DALYs. This was also higher than the burden of 31 selected infectious diseases in the EU/EEA, which was estimated to be 273 (95\% UI:249-299) DALYs per 100,000 population using the BCoDE methodology [19]. Comparisons of our estimates of the number of DALYs with those of the Global Burden of Disease (GBD) project must be 


\section{FIGURE 4}

Total annual burden of five types of healthcare-associated infections ${ }^{\mathrm{a}}$ in Germany (left) and EU/EEA (right), stratified by age and sex and within each stratum the (A-B) total number of DALYs and (C-D) DALYs per 100,000 population, Germany, EU/ EEA, 2011-2012

German PPS

A.

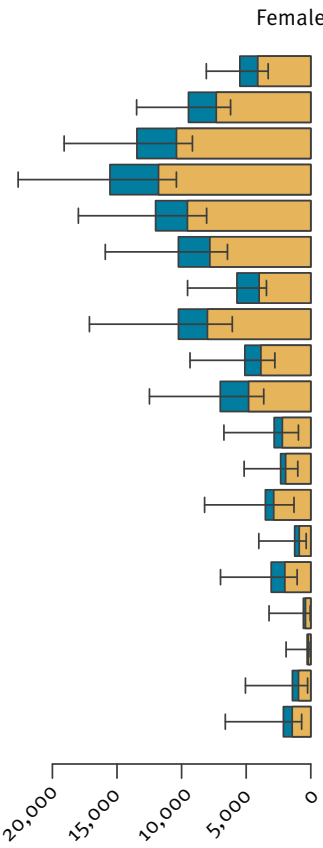

Number of DALYs (all infections)

\section{Male}

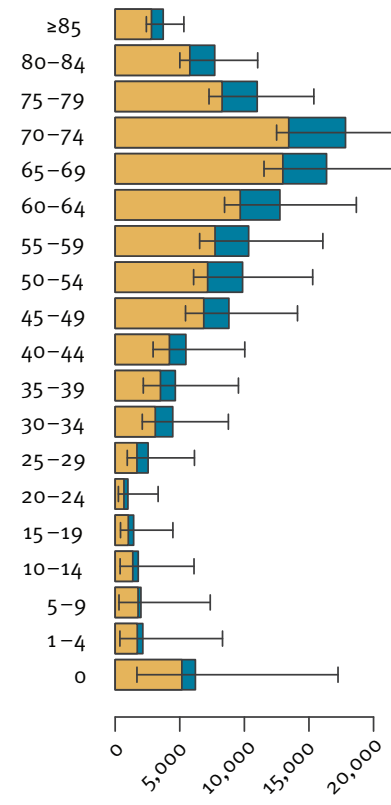

Number of DALYs (all infections)
C.

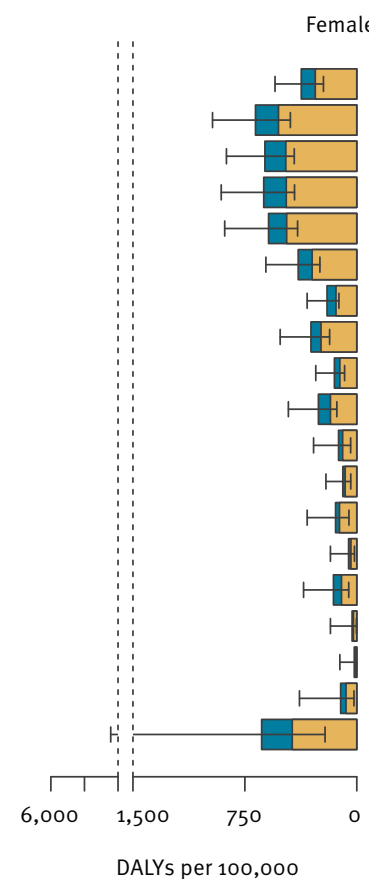

Female Age $^{\mathrm{b}}$ Male

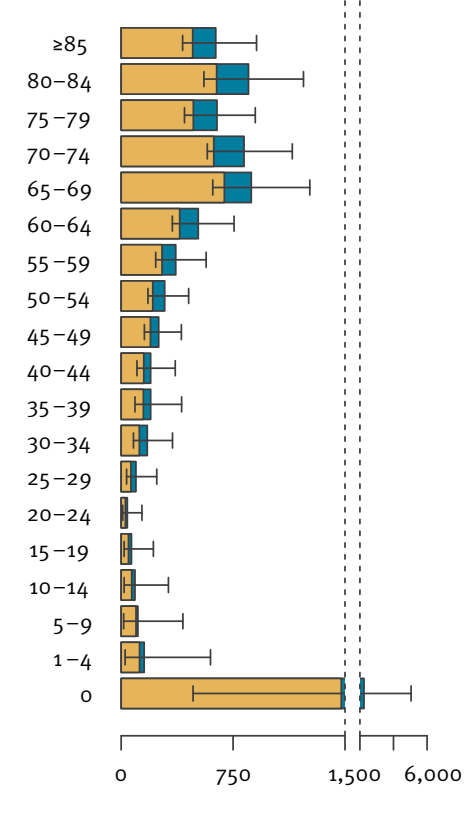

DALYs per 100,000
ECDC PPS (EU/EEA)

B.

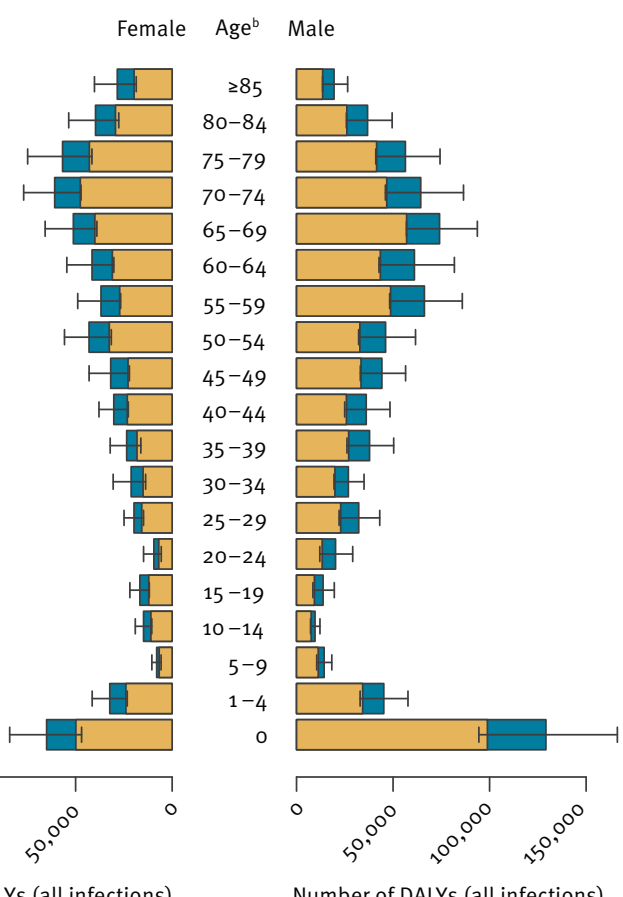

D.

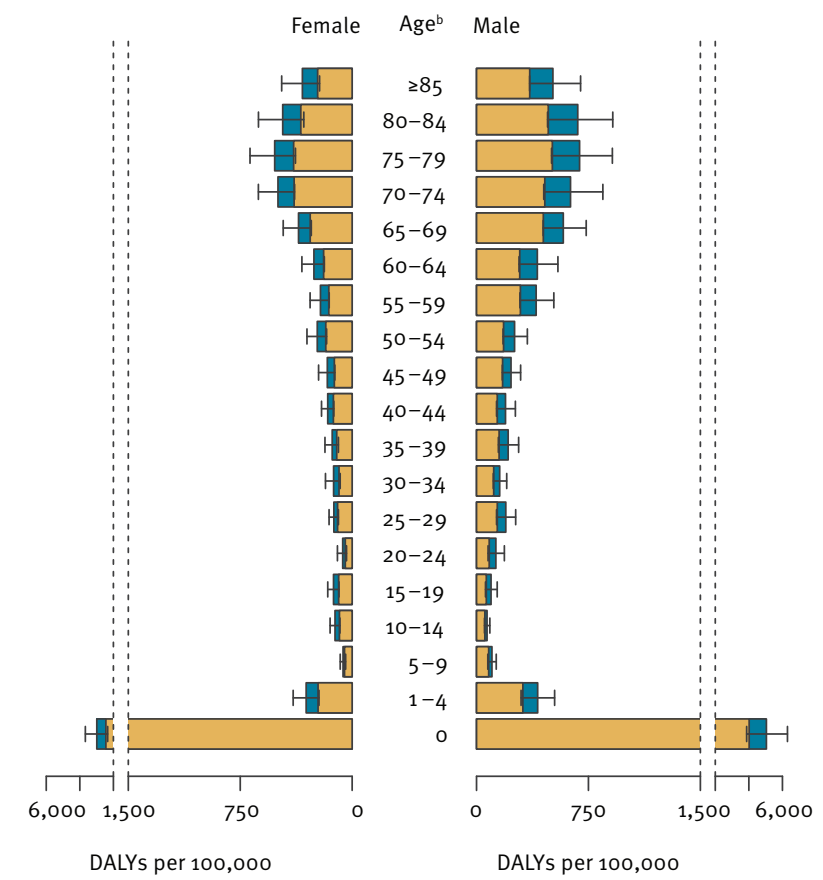

DALY: disability-adjusted life year; ECDC: European Centre for Disease Control and Prevention; EU/EEA: European Union/European Economic Area; PPS: point prevalence survey.

a The five healthcare-associated infections comprise healthcare-associated Clostridioides difficile infection, healthcare-associated pneumonia, healthcare-associated primary bloodstream infection, healthcare-associated urinary tract infection and surgical site infection.

${ }^{b}$ Age in years.

The error bars indicate the $95 \%$ uncertainty intervals. 
conducted with caution, since the latter are derived using a prevalence-based approach [20]. In addition, the burden of HAls is not presented within the GBD project, but is instead attributed to the underlying disease. However, a relative and rough comparison of the burden of HAls with GBD estimates for other diseases is presented in the Supplementary Table $S_{4}[20,21]$.

\section{Comparison of Germany and the European Union/European Economic Area}

To ensure comparability, we estimated the burden of the five types of HAls in the EU/EEA with the same $\mathrm{BHAl}$ methodology and $\mathrm{R}$ package. The number of HAls, attributable deaths and DALYs (YLDs and YLLs) per 100,000 population was overall higher in Germany than in the EU/EEA average (Table 2, Supplementary Table $\mathrm{S}_{1}$ and $\mathrm{S}_{3}$, Figure 3, Supplementary Figure $\mathrm{S}_{5}$ and S6). Since the hospital prevalence of HAls in Germany in 2011 was considerably lower than the EU/EEA average from the ECDC PPS between 2011 and 2012, we initially expected a lower health burden of HAls in Germany [6]. However, extrapolating from the hospitalised to the general population (see Methods, workflow step 3-5), the burden of HAls in Germany was higher than the EU/EEA average. Burden of disease is a measure based on the general population and thus the number of hospitalised patients - which extrapolates the incidence per patient to the general population - is a major factor influencing the burden of HAls in the general population. Germany has the highest number of curative beds in Europe and the second largest number of hospitalised patients per 1,000 population among 34 Organisation for Economic Co-operation and Development (OECD) countries [2224]. Hygiene and infection prevention and control measures affect HAl occurrence among hospitalised patients. Germany's relatively low prevalence among hospitalised patients may be partly explained by the generally good hygiene and infection prevention and control measures in German hospitals. However, since there are so many hospitalised patients, their effectiveness may be diluted when calculating the burden of HAls per general population. This becomes clear when comparing the incidence within the hospitalised population to the incidence per general population. In accordance with $\mathrm{HAl}$ prevalence, the incidence of HAls within the hospitalised population was lower for Germany - 2.40 (95\% UI: 2.12-2.70) per 100 patients than for the EU/EEA - 2.83 (95\% UI: 2.75-2.90) per 100 patients (data not shown). To calculate the incidence per general population, the incidence per patient was multiplied by the number of hospital discharges and normalised by the total population. This results in a higher incidence per general population for Germany - 592.1 (95\%UI:521.7-665.8) per 100,000 population - in comparison to the incidence per general population in the EU/EEA - 467.9 (95\%UI: 456.2-480.2) per 100,000 population. This demonstrates that the number of hospital stays is an important factor, which needs to be considered, alongside other measures, in order to reduce the burden of HAls per general population.
The results of the German and ECDC PPS 2016 indicate that the HAI prevalence may have slightly decreased in Germany since 2011 [25]. Nevertheless, the number of HAls per 100,000 was still estimated to be higher in Germany - 735.6 (95\% Cl: 452.8-1,141.9) - than the EU/ EEA estimate -658.5 (95\% Cl: 437.0-957.6) [13,15].

The burden of UTIS and $C$. difficile was higher in Germany than the EU/EEA average, whereas the burden of HAP was lower in Germany (Figure 3, Supplementary Figure $\mathrm{S}_{5}$ ). This is in line with the ECDC PPS between 2011 and 2012, where Germany was among the countries with the highest proportions of UTIs and $C$. difficile infections among all HAls within the PPS between 2011 and 2012. Since then, efforts have been made to enhance antibiotic stewardship and recommendations have been put in place in order to prevent catheter associated urinary tract infections [26]. The prevalence of UTIs was lower in the PPS 2016 and its proportion among all HAls decreased, whereas prevalence of $C$. difficile infections in Germany increased [27]. Further research is needed to analyse whether these differences point towards more or less effective strategies to prevent these types of HAls.

\section{Comparison of the $\mathrm{BHAI}$ and $\mathrm{BCoDE}$ methodologies}

In principle, the BHAI methodology and the application of BCoDE for HAls as in Cassini et al. [3] have the same approach: prevalence is converted to incidence per patient, which is then extrapolated to the population. Using Monte Carlo simulations, the number of HAls is sampled and identical disease outcome trees are used to estimate the attributable burden of HAls.

However, Cassini et al. used the median of $\mathrm{LOI}_{\mathrm{PPS}}$ to estimate LOI for prevalence to incidence conversion, while BHAl uses a new method that was shown to be less prone to bias and performed more reliably in simulation studies [14].

Moreover, in our analyses we found that the stratified sampling approach in BCoDE $[3,7]$, may overestimate the number of HAls in strata with zero cases in the PPS, which became more frequent with smaller sample size in the range of those in the PPS conducted for individual countries. To address this, BHAI samples the number of HAls in each simulation on the population level and then distributes cases to strata by an age and sex distribution. We carried out simulations using subsamples of the full ECDC PPS dataset between 2011 and 2012 to demonstrate that this methodological adjustment addressed the issue of overestimation in smaller samples (Methods, Supplementary Figure S7). Therefore, we recommend the use of BHAl in future efforts to estimate the health burden of HAls in order to ensure comparability, particularly at the country level.

The new sampling approach in BHAI makes application to data collected with both the ECDC PPS patientbased ('standard') and unit-based('light') protocols 
possible, whereas $B C o D E$ can only be applied to the patient-based protocol. In contrast to the patient-based protocol, data for patients without HAls are not collected with the unit-based protocol. Data on patients without HAls are needed as denominator data for stratification in the original BCoDE approach, but not required for the BHAI methodology. Nevertheless, one limitation of the unit-based protocol is that the mean LA of patients is not recorded, which meant that we had to use hospital denominator data from the PPS. We therefore calculated LA from data of participating hospitals, based on the number of discharges and patient days from the previous year. However, in the patient-based protocol, the mean LA is recorded for each patient. In this case we suggest that LA and LOI are both calculated using the method by Willrich et al., since possible biases or errors in the estimation might cancel out in the division of LA by LOI during the conversion from prevalence to incidence [14].

\section{Limitations}

The available evidence for transition probabilities for the outcome trees was derived from systematic reviews. Even though this may be the most transparent and unbiased approach to design outcome trees, it has to be noted that the underlying literature was sparse and of moderate to low quality [9]. With increasing evidence from the literature, outcome trees will improve and therefore should be reevaluated in the future. Transition probabilities in the outcome trees represent the likelihood of sequelae or death attributable to an infection. This justifies that the burden estimation is based on the number of HAls, and not on the number of patients. Recurrent infections for each patient are implicitly reflected in the transition probabilities. By considering HAls independently, we do not account for the fact that some patients have multiple HAls. In addition, the probability of sequelae, recovery and death might not be independent. But in the German PPS sample, only $7 \%$ (28 patients with two or more HAls/394 patients with one or more HAls) of patients with a HAI had more than one HAI on the day of the PPS. Thus, the violation of the independence assumption likely results in minor overestimation.

We used the European McCabe score distribution for our estimation. The large number of acute care hospital beds in Germany might lead to avoidable hospital stays and a hospitalised patient population with less severe primary diseases than in other EU/EEA countries [22-24]. The European McCabe scores might therefore overestimate the severity of underlying diseases of German hospitalised patients. In turn, DALYs would be underestimated for Germany since the European McCabe distribution would assume lower remaining life expectancies.

Following the study of Cassini et al. we used hospital discharges of 2011 for all countries, although some countries conducted the PPS in 2012 [3]. In addition, LA was calculated using data from the previous year of the participating hospitals. This should not have significantly biased our results since the variation of LA and the number of discharges during the study period was low [13].

Another possible limitation of our study is the representativeness of the PPS data. Although hospital size was the only variable used for randomly sampling hospitals to obtain a representative sample of German hospitals, case mix and specialisation also influence HAI prevalence. For this reason, we also estimated the burden of HAls from the larger convenience sample of German hospitals. This analysis led to higher estimates of the burden of HAls, suggesting that a high uncertainty remains when estimating the burden of HAls from PPS samples.

\section{Conclusion}

In summary, the open-source BHAI R package, was applied to German and EU/EEA data to calculate the burden of HAls at country level, and is applicable to other countries in or outside of the EU/EEA. In Germany, the burden of HAls is higher than the burden of other communicable diseases. Despite the fact that Germany has a relatively low prevalence of HAls compared with other European countries, the burden of HAls in Germany is higher than the EU/EEA average [6]. A probable cause for the high burden of HAls in Germany is the country's large hospital patient population. It has been argued that the large numbers of acute care beds in Germany may lead to avoidable hospital stays [28]. Therefore, the reduction of avoidable hospital stays together with further focus on hygiene measures and infection prevention and control are important steps to reduce the burden of HAls in Germany.

\section{Acknowledgements}

We thank Dominique L. Monnet, European Centre for Disease Prevention and Control, Stockholm, Sweden and Thomas Harder, Robert Koch Institute, Berlin, Germany for their invaluable help and expertise.

\section{Conflict of interest}

None declared.

Authors' contributions

Conceived the project: BZ, SH, TE. Analysed the data: BZ, $\mathrm{SH}$. Wrote the first draft of the manuscript: BZ, SH, TE. Contributed to the prevalence to incidence conversion: NW, JW. Provided and helped with analysis of EU/EEA PPS data: AC, DP, CS. Provided and helped with analysis of the German PPS data: MB, PG. Contributed to interpretation of the results: $A C, D P, C S, M A S, L H W, P G, M B$. Contributed to the writing of the manuscript: all authors. ICMJE criteria for authorship read and met: all authors. Agree with manuscript results and conclusions: all authors. 


\section{References}

1. Gastmeier P, Brunkhorst F, Schrappe M, Kern W, Geffers C. [How many nosocomial infections are avoidable?]. Dtsch Med Wochenschr. 2010;135(3):91-3. https://doi org/10.1055/s-0029-1244823 PMID: 20077383

2. Zingg W, Holmes A, Dettenkofer M, Goetting T, Secci F, Clack $\mathrm{L}$, et al. systematic review and evidence-based guidance on organization of hospital infection control programmes(SIGHT) study group. Hospital organisation, management, and structure for prevention of health-care-associated infection: a systematic review and expert consensus. Lancet Infect Dis. 2015;15(2):212-24. https://doi.org/10.1016/S14733099(14)70854-0 PMID: 25467650

3. Cassini A, Plachouras D, Eckmanns T, Abu Sin M, Blank HP, Ducomble T, et al. Burden of Six Healthcare-Associated Infections on European Population Health: Estimating Incidence-Based Disability-Adjusted Life Years through a Population Prevalence-Based Modelling Study. PLoS Med. 2016;13(10):e1002150. https://doi.org/10.1371/journal. pmed.1002150 PMID: 27755545

4. Kretzschmar $M$, Mangen $M J$, Pinheiro $P$, Jahn $B$, Fèvre EM, Longhi S, et al. BCoDE consortium. New methodology for estimating the burden of infectious diseases in Europe. PLoS Med. 2012;9(4):e1001205. https://doi.org/10.1371/journal. pmed.1001205 PMID: 22529750

5. Mangen MJ, Plass D, Havelaar AH, Gibbons CL, Cassini A, Mühlberger N, et al. BCoDE consortium. The pathogen- and incidence-based DALY approach: an appropriate [corrected] methodology for estimating the burden of infectious diseases. PLoS One. 2013;8(11):e79740. https://doi.org/10.1371/journal. pone.0079740 PMID: 24278167

6. European Centre for Disease Control and Prevention(ECDC). Point prevalence survey of healthcare-associated infections and antimicrobial use in European acute care hospitals. Stockholm: ECDC; 2013.

7. Colzani E, Cassini A, Lewandowski D, Mangen MJ, Plass D, McDonald SA, et al. A Software Tool for Estimation of Burden of Infectious Diseases in Europe Using Incidence-Based Disability Adjusted Life Years. PLoS One. 2017;12(1):e0170662. https:// doi.org/10.1371/journal.pone.0170662 PMID: 28107447

8. European Centre for Disease Control and Prevention (ECDC). Point prevalence survey of healthcare-associated infections and antimicrobial use in European acute care hospitals protocol version 4.3. Stockholm: ECDC; 2012.

9. Abu Sin M, Blank HP, Ducomble T, Eckmanns T, Haller S, Harder $\mathrm{T}$, et al. Burden of healthcare associated infection (BHAI)evidence-based and comorbidity-adjusted outcome trees for estimation of burden of disease. Berlin: Robert Koch-Institut; 2016.

10. Haller S, Deindl P, Cassini A, Suetens C, Zingg W, Abu Sin $M$, et al. Neurological sequelae of healthcare-associated sepsis in very-low-birthweight infants: Umbrella review and evidence-based outcome tree. Euro Surveill. 2016;21(8):30143. https://doi.org/10.2807/1560-7917.ES.2016.21.8.30143 PMID: 26940884

11. Haagsma JA, Maertens de Noordhout C, Polinder S, Vos T, Havelaar AH, Cassini A, et al. Assessing disability weights based on the responses of 30,660 people from four European countries. Popul Health Metr. 2015;13(1):10. https://doi. org/10.1186/s12963-015-0042-4 PMID: 26778920

12. Rhame FS, Sudderth WD. Incidence and prevalence as used in the analysis of the occurrence of nosocomial infections. Am J Epidemiol. 1981;113(1):1-11. https://doi.org/10.1093/ oxfordjournals.aje.a113058 PMID: 7457475

13. Eurostat - Hospital discharges and length of stay for inpatient and curative care. Available from: http://appsso.eurostat. ec.europa.eu/nui/show.do?dataset=hlth_co_dischls\&lang=en

14. Willrich N, Haller S, Eckmanns T, Zacher B, Kärki T, Plachouras $\mathrm{D}$, et al. From prevalence to incidence - a new approach in the nosocomial setting. bioRxiv. 2019:554725.

15. Suetens C, Latour K, Kärki T, Ricchizzi E, Kinross P, Moro ML, et al. The Healthcare-Associated Infections Prevalence Study Group. Prevalence of healthcare-associated infections, estimated incidence and composite antimicrobial resistance index in acute care hospitals and long-term care facilities: results from two European point prevalence surveys, 2016 to 2017. Euro Surveill. 2018;23(46):1800516. https://doi. org/10.2807/1560-7917.ES.2018.23.46.1800516 PMID: 30458912

16. Plass D, Mangen MJ, Kraemer A, Pinheiro P, Gilsdorf A, Krause $G$, et al. The disease burden of hepatitis $B$, influenza, measles and salmonellosis in Germany: first results of the burden of communicable diseases in Europe study. Epidemiol Infect. 2014;142(10):2024-35. https://doi.org/10.1017/ So950268813003312 PMID: 24480146
17. Rüden HDF, Schumacher M. Nosokomiale Infektionen in Deutschland - Erfassung und Prävention (NIDEP-Studie). [Nosocomial infections in Germany - detection and prevention (NIDEP study)]. In: Gesundheit BdSdBf, 1st edition. BadenBaden: Nomos Verlagsgesellschaft; 1995. German.

18. Gastmeier P, Geffers C. [Nosocomial infections in Germany. What are the numbers, based on the estimates for 2006?] Dtsch Med Wochenschr. 2008;133(21):1111-5. https://doi. org/10.1055/s-2008-1077224 PMID: 18478503

19. Cassini A, Colzani E, Pini A, Mangen MJ, Plass D, McDonald SA, et al. On Behalf of The BCoDE Consortium. Impact of infectious diseases on population health using incidence-based disability-adjusted life years (DALYs): results from the Burden of Communicable Diseases in Europe study, European Union and European Economic Area countries, 2009 to 2013. Euro Surveill. 2018;23(16):1700454. https://doi.org/10.2807/15607917.ES.2018.23.16.17-00454 PMID: 29692315

20. Murray CJ, Ezzati M, Flaxman AD, Lim S, Lozano R, Michaud C, et al. GBD 2010: a multi-investigator collaboration for global comparative descriptive epidemiology. Lancet. 2012;38o(9859):2055-8. https://doi.org/10.1016/S01406736(12)62134-5 PMID: 23245598

21. Plass D, Vos T, Hornberg C, Scheidt-Nave C, Zeeb H, Krämer A. Trends in disease burden in Germany: results, implications and limitations of the Global Burden of Disease study. Dtsch Arztebl Int. 2014;111(38):629-38. PMID: 25316518

22. Eurostat - Number of hospital beds (curative care). Available from: http://ec.europa.eu/eurostat/tgm/table.do?tab=table\&in it $=1 \&$ language $=$ de $\&$ pode $=$ tps00168\&plugin $=1$

23. Kumar A, Schoenstein M. Managing Hospital Volumes: Germany and Experiences from OECD Countries. Paris: Organization for Economic Cooperation and Development (OECD); 2013.

24. Rhodes A, Ferdinande P, Flaatten H, Guidet B, Metnitz PG, Moreno RP. The variability of critical care bed numbers in Europe. Intensive Care Med. 2012;38(10):1647-53. https://doi. org/10.1007/s00134-012-2627-8 PMID: 22777516

25. Deutsche nationale Punkt-Prävalenzerhebung zu nosokomialen Infektionen und Antibiotika-Anwendung 2016. [German national point prevalence survey on nosocomial infections and antibiotic use 2016]. Nationalen Referenzzentrums für Surveillance von nosokomialen Infektionen; 2017. German. Available from: https://www.nrz-hygiene.de/fileadmin/nrz/ download/pps2016/PPS_2016_Abschlussbericht_20.07.2017. pdf

26. KRINKO. Prävention und Kontrolle Katheter-assoziierter Harnwegsinfektionen: Empfehlung der Kommission für Krankenhaushygiene und Infektionsprävention (KRINKO) beim Robert Koch-Institut. Bundesgesundheitsblatt Gesundheitsforschung Gesundheitsschutz. 2015;58(6):641-50. https://doi.org/10.1007/s00103-015-2152-3 PMID: 25824136

27. Behnke M, Aghdassi SJ, Hansen S, Diaz LAP, Gastmeier P, Piening B. The Prevalence of Nosocomial Infection and Antibiotic Use in German Hospitals. Dtsch Arztebl Int. 2017;114(50):851-7. https://doi.org/10.3238/arztebl.2017.0851 PMID: 29271343

28. Busse R, Ganten D, Huster S, Reinhardt ER, Suttorp N, Wiesing U. Zum Verhältnis von Medizin und Ökonomie im deutschen Gesundheitssystem. [On the relationship between medicine and economics in the German health system]. Halle (Saale): Deutsche Akademie der Naturforscher Leopoldina e.V.; 2016. German

\section{License, supplementary material and copyright}

This is an open-access article distributed under the terms of the Creative Commons Attribution (CC BY 4.0) Licence. You may share and adapt the material, but must give appropriate credit to the source, provide a link to the licence and indicate if changes were made.

Any supplementary material referenced in the article can be found in the online version.

This article is copyright of the authors or their affiliated institutions, 2019. 